\title{
ORDER-CUSHIONED REFINEMENTS AND NORMALITY
}

\author{
J. C. SMITH AND RASTISLAV TELGARSKY
}

\begin{abstract}
The authors use the notions of order-cushioned covers and weak $\bar{\theta}$-covers to obtain the following result.

THEOREM. A space $X$ is collectionwise normal iff every weak $\bar{\theta}$-cover of $X$ has an order-cushioned open refinement.

Similar characterizations are obtained for normal, countably paracompact spaces and analogous embedding theorems are shown.
\end{abstract}

\section{Introduction.}

DEFINITION 1.1. Let $U=\left\{U_{\alpha}: \alpha \in A\right\}$ be a collection of subsets of a space $X$ and let $\leq$ be a linear order on $A$.

The collection $\mathcal{U}$ is order-cushioned in $\mathcal{V}$ with cushion map $f: \mathcal{U} \rightarrow \mathcal{V}$ provided, for every $U_{\alpha} \in \mathcal{U}$, every $x \in U_{\alpha}$ and every $B \subseteq\{\beta: \beta \leq \alpha\}$, if $x \in \overline{\bigcup_{\beta \in B} U_{\beta}}$, then $x \in \bigcup_{\beta \in B}\left\{\left(U_{\beta}\right)\right.$. In 1967 Katuta [4] introduced the notion of an order locally finite collection and obtained the following results.

THEOREM 1.2. A regular space is paracompact iff every open cover of $X$ has an order locally finite open refinement.

Recently several authors $[6,8,9,15,16]$ have studied the analogous notions of order closure-preserving and order-cushioned refinements. In particular, Vaughan [16] has shown that Theorem 1.2 above fails to be true when order locally finite is replaced by order-cushioned, yet remains true when order locally finite is replaced by linearly cushioned.

Martin [6], on the other hand, has established the following interesting result.

THEOREM 1.3. If $X$ is a regular space such that every open cover of $X$ has an order-cushioned open refinement, then $X$ is collectionwise normal and countably paracompact.

In this paper we obtain results which are analogous to the above theorem of Martin. In $\S 2$ we obtain a rather nice and unusual cover characterization of collectionwise normal spaces using the notion of weak $\bar{\theta}$-covers. Analogous characterizations for normal, countably paracompact spaces are obtained in $\S 3$, while embedding characterizations are briefly discussed in $\S 4$. Several open questions are also included. The following are included for the benefit of the reader.

DEFINITION 1.4. A space $X$ is called weak $\bar{\theta}$-refinable if every open cover of $X$ has a refinement $\bigcup_{i=1}^{\infty} \mathcal{G}_{i}$ satisfying:

(i) each $\mathcal{G}_{i}=\left\{G(\alpha, i): \alpha \in A_{i}\right\}$ is a collection of open subsets of $X$;

Received by the editors November 10, 1980 and, in revised form, October 9, 1981.

1980 Mathematics Subject Classification. Primary 54D20, 54D15; Secondary 54A25, 54D30.

Key words and phrases. Paracompact, collectionwise normal, normal, countably paracompact, weak $\bar{\theta}$-cover, order locally finite, order closure-preserving, order-cushioned, locally finite, point finite, star-countable, shrinkable, cushioned, embedded. 
(ii) for each $x \in X, 0<\operatorname{ord}\left(x, \mathcal{G}_{k}\right)<\infty$ for some $k$;

(iii) the open cover $\left\{G_{i}^{*}=\bigcup\left\{G: G \in \mathcal{G}_{i}\right\}\right\}_{i=1}^{\infty}$ is point finite.

An open cover which satisfies properties (i)-(iii) is called a weak $\bar{\theta}$-cover.

In [9] the author has shown the following.

THEOREM 1.5. Let $X$ be a normal space. Then $X$ is collectionwise normal iff every weak $\bar{\theta}$-cover of $X$ has a locally finite open refinement.

We now generalize this result.

2. Collectionwise normal spaces. In [8] the author obtained the following open cover characterization for the class of collectionwise normal spaces.

THEOREM 2.1. Let $X$ be a normal space. Then the following conditions are equivalent:

(1) is a collectionwise normal.

(2) Every weak $\bar{\theta}$-cover of $X$ has an order locally finite open refinement.

(3) Every weak $\bar{\theta}$-cover of $X$ has a precise order closure-preserving open refinement.

(4) Every weak $\bar{\theta}$-cover of $X$ has a precise order-cushioned open refinement.

(5) Every weak $\bar{\theta}$-cover of $X$ has a well-ordered closure-preserving open refinement.

(6) Every weak $\bar{\theta}$-cover of $X$ has a well-ordered cushioned open refinement.

In fact in [8] questions were raised as to (1) whether the "precise" or "wellordered" conditions in Theorem 2.1 could be removed, and (2) were there similar characterizations without assuming normality? We now answer both of these questions in the affirmative. The proof is similar to that for Lemma 2.2 of [6].

THEOREM 2.2. A space $X$ is collectionwise normal iff every weak $\bar{\theta}$-cover of $X$ has an order-cushioned open refinement.

Proof. (NeCESSITY) Let $X$ be collectionwise normal. From Theorem 1.5 above every weak $\bar{\theta}$-cover $\mathcal{G}$ of $X$ has a locally finite open refinement. Since $X$ is normal, locally finite open covers are shrinkable by Theorem 1.2 of $[\mathbf{1 0}]$ and hence $\mathcal{G}$ has a locally finite open refinement $\nLeftarrow$ such that $\bar{H}$ also refines $\mathcal{G}$. It is easy to see that $\mathcal{H}$ is order-cushioned in $\mathcal{G}$.

(SUFFICIENCY) Let $\left\{F_{s}: s \in S\right\}$ be a discrete collection of closed subsets of $X$. Define $G_{s}=X-\bigcup_{s^{\prime} \neq s} F_{s^{\prime}}$ for each $s \in S$ and $G_{0}=X-\bigcup_{s \in S} F_{s}$. Clearly $\mathcal{G}=\left\{G_{0}\right\} \cup\left\{G_{s}: s \in S\right\}$ is a weak $\bar{\theta}$-cover of $X$. Let $\mathcal{H}=\left\{H_{\alpha}: \alpha \in(A,<)\right\}$ be an open order-cushioned refinement of $\mathcal{G}$ with cushioned map $\Phi: \not H \rightarrow \mathcal{G}$.

Let $s \in S$. If $H_{\alpha} \in \mathcal{H}$ such that $H_{\alpha} \cap F_{s} \neq \emptyset$, define $U(\alpha, s)=H_{\alpha}$ $\bigcup\left\{H_{\beta}: \beta<\alpha: \phi\left(H_{\beta}\right) \cap F_{s}=\emptyset\right\}$, for each $\alpha \in A$ and let

$$
U_{s}=\bigcup\left\{U(\alpha, s): U(\alpha, s) \cap F_{s} \neq \emptyset\right\} .
$$

If $\left\{H_{\alpha} \cap F_{s}=\emptyset\right\}$, define $U(\alpha, s)=\emptyset$. We assert that $\left\{U_{s}: s \in S\right\}$ is the desired disjoint system of neighborhoods of $\left\{F_{s}: s \in S\right\}$.

(1) Suppose $s \neq s^{\prime}$. Choose $H_{\alpha}, H_{\beta} \in \not H$ such that $H_{\alpha} \cap F_{s} \neq \emptyset$ and $H_{\beta} \cap F_{s^{\prime}} \neq$ $\emptyset$. Since $\nvdash<\mathcal{G}, \alpha \neq \beta$; so assume $\beta<\alpha$. Since $H_{\beta} \cap F_{s}=\emptyset$, then $\phi\left(H_{\beta}\right) \cap F_{s}=$ $\emptyset$, so that $H_{\beta} \cap U(\alpha, s)=\emptyset$. But $U\left(\beta, s^{\prime}\right) \subseteq H_{\beta}$, and hence, $U(\alpha, s) \cap U\left(\beta, s^{\prime}\right)=\emptyset$. Therefore $U_{s} \cap U_{s^{\prime}}=\emptyset$. 
(2) Suppose $x \in F_{s}$. We show that $x \in U_{s}$ and hence $F_{s} \subseteq U_{s}$. If $x \notin U_{s}$ then $x \notin U(\alpha, s)$ for each $\alpha \in A$. Choose $\alpha_{0} \in A$ such that $x \in H_{\alpha_{0}} \cap F_{s}$. Since

$$
x \notin U\left(\alpha_{0}, s\right)=H_{\alpha_{0}}-\overline{\bigcup\left\{H_{\beta}: \beta<\alpha_{0}: \phi\left(H_{\beta}\right) \cap F_{s}=\emptyset\right\}},
$$

then $x \in \overline{\bigcup\left\{H_{\beta}: \beta<\alpha_{0}: \phi\left(H_{\beta}\right) \cap F_{s}=\emptyset\right\}}$. But $\not H$ is order-cushioned in $\mathcal{G}$ so that $x \in \Phi\left(H_{\beta}\right)$ for some $\beta<\alpha_{0}$ so that $\phi\left(H_{\beta}\right) \cap F_{s}=\emptyset$. However $x \in \phi\left(H_{\beta}\right) \cap F_{s}$, a contradiction.

REMARK. It is interesting here to note that $(1) \equiv(6)$ in Theorem 2.1 above remains true not only when the "well-ordered" and "precise" conditions are dropped but also when "normality" is removed.

3. Normal and countably paracompact spaces. From the proof of Theorem 2.2 above we now observe the following.

THEOREM 3.1. If every countable (or finite) open cover of a space $X$ has an order-cushioned open refinement, then $X$ is normal.

PROOF. The proof follows from Theorem 2.2 above where $|S| \leq \aleph_{0}$.

Clearly, if $X$ is normal and countably paracompact then every countable open cover of $X$ has an open locally finite refinement whose closures also refine. Therefore countable open covers have well-order-cushioned open refinements. It is natural to suspect therefore that this condition is necessary and sufficient for a space to be normal and countably paracompact.

THEOREM 3.2. If every countable open cover of a space $X$ has a well-ordercushioned open refinement, then $X$ is countably metacompact.

ProOF. Let $\left\{F_{i}\right\}_{i=1}^{\infty}$ be a decreasing sequence of closed subsets of $X$ such that $\bigcap_{i=1}^{\infty} F_{i}=\emptyset$. Now $\left\{G_{i}=X-F_{i}\right\}_{i=1}^{\infty}$ is a countable open cover of $X$ and hence has a well-order-cushioned open refinement $\nLeftarrow=\left\{H_{\alpha}: \alpha \in(A, \leq)\right\}$ with cushion map $\Phi$. Now for each $i$ and each $\alpha \in A$, we define

$$
U_{\alpha}\left(F_{i}\right)=\left\{\begin{array}{l}
H_{\alpha}-\overline{U\left\{H_{\beta}: \beta<\alpha, \phi\left(H_{\beta}\right) \cap F_{i}=\phi\right\}}, \quad H_{\alpha} \cap F_{i} \neq \emptyset \\
\emptyset, \quad H_{\alpha} \cap F_{i}=\emptyset
\end{array}\right\}
$$

and let $U_{i}=\bigcup_{\alpha \in A} U_{\alpha}\left(F_{i}\right)$.

We assert that (1) $F_{i} \subseteq U_{i}$ for each $i$, and (2) $\bigcap_{i=1}^{\infty} U_{i}=\emptyset$; so that $X$ is countably metacompact.

(1) Let $x \in F_{i}$. Then there exists some $\alpha \in A$ such that $x \in H_{\alpha} \cap F_{i}$. Now

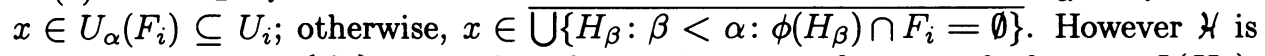
order-cushioned in $\left\{G_{i}\right\}_{i=1}^{\infty}$ so that there exists some $\beta<\alpha$ such that $x \in \Phi\left(H_{\beta}\right)$, where $\phi\left(H_{\beta}\right) \cap F_{i}=\emptyset$. This is a contradiction.

(2) Suppose there exists a point $y \in \bigcap_{i=1}^{\infty} U_{i}$. It is easy to show that there exists an increasing sequence $\{n(i)\}_{i=1}^{\infty}$ such that $y \in U_{\alpha_{n(i)}}\left(F_{n(i)}\right) \subseteq H_{\alpha_{n(i)}}$ where $H_{\alpha_{n(i)}} \cap F_{n(i)} \neq \emptyset$ and $\phi\left(H_{\alpha_{n(i)}}\right) \cap F_{n(i+1)}=\emptyset$.

Since $(A, \leq)$ is well-ordered there exists a first element $\alpha_{n\left(i_{0}\right)} \in\left\{\alpha_{n(i)}: i=\right.$ $1,2, \ldots\}$. Now choose $i>i_{0}$ so that $\alpha_{n\left(i_{0}\right)}<\alpha_{n(i)}$. However,

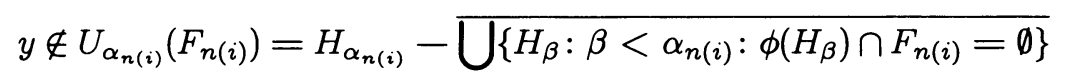

since $y \in H_{\alpha_{n\left(i_{0}\right)}}$ and $\phi\left(H_{\alpha_{n\left(i_{0}\right)}}\right) \cap F_{n(i)}=\emptyset$ as $\alpha_{n\left(i_{0}\right)}<\alpha_{n(i)}$. This is a contradiction and the result is proved. 
COROLlARY 3.3. A space $X$ is normal and countably paracompact iff every countable open cover has a well-order-cushioned open refinement.

We can now state a generalization to a theorem of Martin [6].

COROLlARY 3.4. Let $X$ be a topological space. Then $X$ is collectionwise normal and countable paracompact iff every weak $\bar{\theta}$-cover of $X$ has an order-cushioned open refinement and every countable open cover of $X$ has a well-order-cushioned open refinement.

REMARK. The referee has pointed out to the authors that Theorem 3.2 is not true if the "well-order-cushioned" is replaced by "order-cushioned". The reason is that any collection $\left\{G_{\alpha}: \alpha \in(A, \leq)\right\}$ is order-cushioned in itself if $\left\{G_{\alpha}: \alpha \in\right.$ $(A, \leq)\}$ is decreasing with respect to $(A, \leq)$. Therefore any increasing (countable) collection is order-cushioned in itself if the reverse order on $A$ is used as the linear order.

4. Some embedding characterizations and open questions. In [1, 2, 7] Alo and Shapiro and in [12] Nichols and Smith have obtained several embedding characterizations for collectionwise normal and expandable spaces. Using these results and the results obtained in the previous sections we now obtain new embedding characterizations for collectionwise normal spaces.

Definition 4.1. Let $S \subseteq X$ and let $\mathcal{G}$ be an open (in $S$ ) cover of $S$. We say that $\mathcal{G}$ has a refinement which can be extended to a cover of $X$ if there exists a cover $\mathcal{U}$ of $X$ such that $\mathcal{U} / S$ refines $\mathcal{G}$.

DEFINITION 4.2. A subset $S \subseteq X$ is said to be $W_{1}$-embedded in $X$ if every locally finite open cover of $S$ has a refinement which can be extended to an ordercushioned open cover of $X$.

DEFINITION 4.3. A subset $S \subseteq X$ is said to be $W_{2}$-embedded in $X$ if every point finite open cover of $S$ has a refinement which can be extended to an order-cushioned open cover of $X$.

REMARK. This notion of embeddedness is a generalization of a subset being strongly $P$-embedded due to Alo and Shapiro [2].

DEFINITION 4.4. A subset $S \subseteq X$ is said to be $W_{3}$-embedded in $X$ if every weak $\bar{\theta}$-cover of $S$ has a refinement which can be extended to an order-cushioned open cover of $X$.

THEOREM 4.5. The following are equivalent for a space $X$.

(1) $X$ is collectionwise normal.

(2) Every closed subset of $X$ is $W_{1}$-embedded in $X$.

(3) Every closed subset of $X$ is $W_{2}$-embedded in $X$.

(4) Every closed subset of $X$ is $W_{3}$-embedded in $X$.

Proof. Clearly (4) $\Rightarrow(3) \Rightarrow(2)$ and the fact that $(2) \Rightarrow(1)$ follows from the same proof as Theorem 2.2 above. We first show that $(1) \Rightarrow(3)$. Let $\mathcal{G}$ be a point finite open cover of $S=\bar{S} \subseteq X$. For each $G \in \mathcal{G}$, let $G^{*}$ be an open subset of $X$ such that $G=G^{*} \cap S$. Define $\mathscr{H}_{1}=\left\{G^{*} ; G \in \mathcal{G}\right\}$ and $\mathscr{H}_{2}=\{X-S\}$. It is easy to see that $\mathcal{H}=\bigcup_{i=1}^{2} H_{i}$ is a weak $\bar{\theta}$-cover of $X$ such that $\mathcal{H} \mid S=\mathcal{G}$. By Theorem 2.2 above, $\forall$ has an order-cushioned open refinement so that $S$ is $W_{2}$-embedded in $X$. 
Finally we show that $(1) \Rightarrow(4)$. Let $\mathcal{G}$ be a weak $\bar{\theta}$-cover of $S=\bar{S} \subseteq X$. By Theorem 2.2 of $[9], \mathcal{G}$ is normal and hence has a locally finite open refinement. Since $(1) \equiv(2), S$ is $W_{3}$-embedded in $X$.

QUESTIONS. (1) Do analogous results hold for order closure-preserving refinements?

(2) What property is equivalent to every order-cushioned (resp. order closurepreserving or order locally finite cover) being shrinkable? Note that if every ordercushioned open cover is shrinkable, then the space is normal and countably paracompact.

(3) Do any of the above results hold when order-cushioned is replaced by $\sigma$-ordercushioned?

(4) What property is equivalent to every open cover having an order-cushioned open refinement?

The authors would like to thank the referee for his comments concerning this paper.

\section{REFERENCES}

1. R. A. Alo and H. L. Shapiro, Paracompact subspaces, Acta Math. Acad. Sci. Hungar 21 (1970), 115-119.

2. __ Countably paracompact, normal and collectionwise normal spaces, Indag. Math. 35 (1973), 346-351.

3. H. R. Bennett and D. J. Lutzer, A note on weak $\theta$-refinability, General Topology Appl. 2 (1972), p. $48-54$.

4. Y. Katuta, $A$ theorem on paracompactness of product spaces, Proc. Japan Acad. 43 (1967), 615-618.

5. L. L. Krajewski, Expanding locally finite collections, Canad. J. Math. 23 (1971), 58-68.

6. H. W. Martin, Linearly ordered covers, normality and paracompactness, Topology Appl. 12 (1981), 305-313.

7. H. L. Shapiro, Extensions of pseudometrics, Canad. J. Math. 18 (1966), 981-998.

8. J. C. Smith, On collectionwise normality, Colloq. Math. 40 (1978), 69-76.

9. , New characterizations for collectionwise normal spaces, Glas. Math. 12 (32) (1977), 327338.

10. __ Applications of shrinkable covers, Proc. Amer. Math. Soc. 73 (1979), 379-387.

11. __ A remark on embeddings and discretely expandable spaces, Proceedings of Topological Symposium (Budapest, Hungary, 1972), Colloq. Math. Soc. János Bolyai 8, Topics in Topology, Keszthely, Hungary, 1972, pp. 575-583.

12. J. C. Smith and J. C. Nichols, Embedding characterizations for expandable spaces, Duke Math. J. 39 (1972), 489-496.

13. J. C. Smith, Properties of $\bar{\theta}$-refinable spaces, Proc. Amer. Math. Soc. 53 (1975), 511-517.

14. J. C. Smith and L. Krajewski, Expandability and collectionwise normality, Trans. Amer. Math. Soc. 160 (1971), 437-451.

15. R. Telgársky, $C$-scattered and paracompact spaces, Fund. Math. 73 (1971), 59-74.

16. J. Vaughan, Linearly ordered collections and paracompactness, Proc. Amer. Math. Soc. 24 (1970), 186-192.

Department of Mathematics, Virginia Polytechnic Institute and State UNIVERSITY, BLACKSBURG, VIRGINIA 24061

Institute of Mathematics, Technical University, Wroclaw, Poland 